\title{
On the Role of the $\mathrm{N}-\mathrm{N}^{+}$Junction Doping Profile of a PIN Diode on Its Turn-Off Transient Behavior
}

\author{
Bruno Allard, Senior Member, IEEE, Hatem Garrab, Tarek ben ben Salah, Herve Morel, Member, IEEE, \\ Kaiçar Ammous, and Kamel Besbes
}

\begin{abstract}
This paper focuses on the role of the $\mathrm{N}-\mathrm{N}^{+}$junction doping profile model of a PiN diode on its turn-off transient and, particularly, the influence of multiple epitaxies in the $\mathrm{N}-\mathrm{N}^{+}$profile. A conventional doping profile model has been used in a previous work and an identification procedure for the main design parameters has been demonstrated. However the validity range of identified PiN-diode models appeared quite limited for hard current and voltage conditions. Readers have asked for the effect of a more advanced doping profile. The turn-off transient of an STTB506D device is considered from experimental and simulation point-ofview inside a fully characterized switching cell. A limitation of the conventional doping profile model is demonstrated and explained physically in order to introduce the necessity of a more complex doping profile. An advanced doping profile is then considered and a comparative study between experimental and simulated turn-off transient behavior of the device is established.
\end{abstract}

Index Terms-PiN diode.

\section{INTRODUCTION}

A CCURATE modeling of the switching behavior of ultrafast power diodes is very important for integrated power converter design, as the transient operations can lead to large voltage and current overshoots, power switching losses, and electromagnetic compatibility (EMC) contributions. Simulation relies on component models and associated parameters. In a previous study [1], it was shown that it is possible to extract good values of the main design parameters of a PiN diode: the doping concentration $\mathrm{N}_{D}$ of the low-doped base region, the effective area A, the width $W_{D}$ of the low-doped base region, and the ambipolar lifetime $\tau$ in the low-doped base region of the PiN diode. This parameter extraction technique is applied to different devices under test.

The diode model with identified parameters [1] yields a very good accuracy between simulation and experimental results for medium current and voltage conditions, as shown in Fig. 1. However for higher current and voltage conditions, the diode model in [1] leads to waveforms that do not match experimental results. Readers have suggested that the diode model was too simple. So the current paper analyzes the cause of the discrepancies between simulation and experience to produce a better PiN diode model. It is considered a modified doping profile in

Manuscript received November 13, 2006; revised January 21, 2007. Recommended for publication by Associate Editor J. Shen

B. Allard, H. Garrab, T. ben Salah, and H. Moreal are with the Ampere Laboratory, UMR CNRS, Villeurbanne F-69621, France (e-mail: bruno.allard@ insalyon.fr).

K. Ammous and K. Besbes are with the Laboratoire de Microélectronique et Instrumentation, Monastir 5000, Tunisia.

Color versions of one or more of the figures in this letter are available online at http://ieeexplore.ieee.org.

Digital Object Identifier 10.1109/TPEL.2007.911882
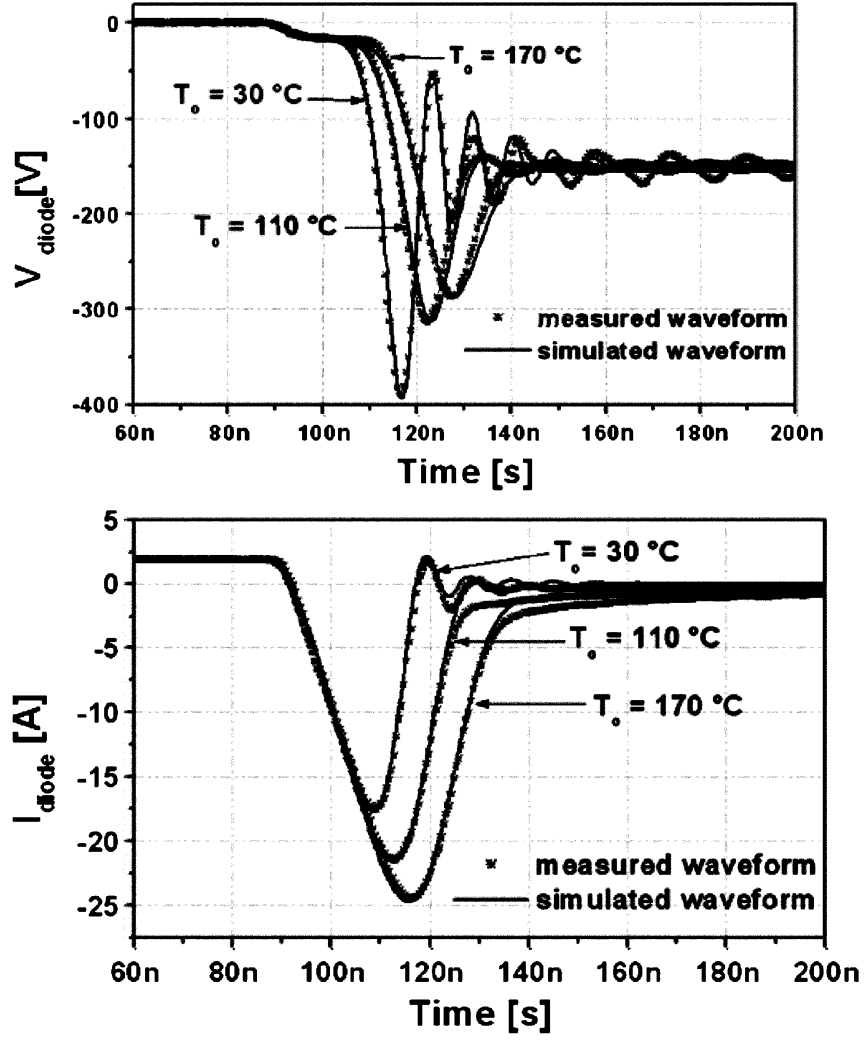

Fig. 1. Comparison of experimental and simulation results at various temperatures during turn-off transient of an STTA 81200 device $\left(I_{F}=2 \mathrm{~A}, V_{R}=\right.$ $160 \mathrm{~V}$ ) [1]. Voltage waveforms (top), current waveforms (bottom).

the diode base region. Pragmatism suggests first considering the simplest modification, as one $\mathrm{N}-\mathrm{N}^{+}$doping step at the extremity of the base region.

Semiconductor manufacturers optimize the extremity of the diode base region, as it is common knowledge that it helps soften the diode reverse recovery [2]. This involves a complex fabrication process with multiple epitaxies. The letter addresses the role, from a modeling point of view, of a double epitaxy that introduces one step at the extremity of the central epitaxial layer. The influence of this step in the $\mathrm{N}-\mathrm{N}^{+}$profile on the turn-off transient behavior of the PiN diode is now detailed. The identification of the additional model parameters related to the doping profile is not addressed, first, because it does not change the procedure detailed in [1], and, second, for the sake of letter length.

\section{Circuit Model of the Switching Cell}

A simple MOSFET transistor/power PiN diode is considered to exhibit turn-off behavior of the diode. The experimental and simulated circuits of the switching cell are pictured in 


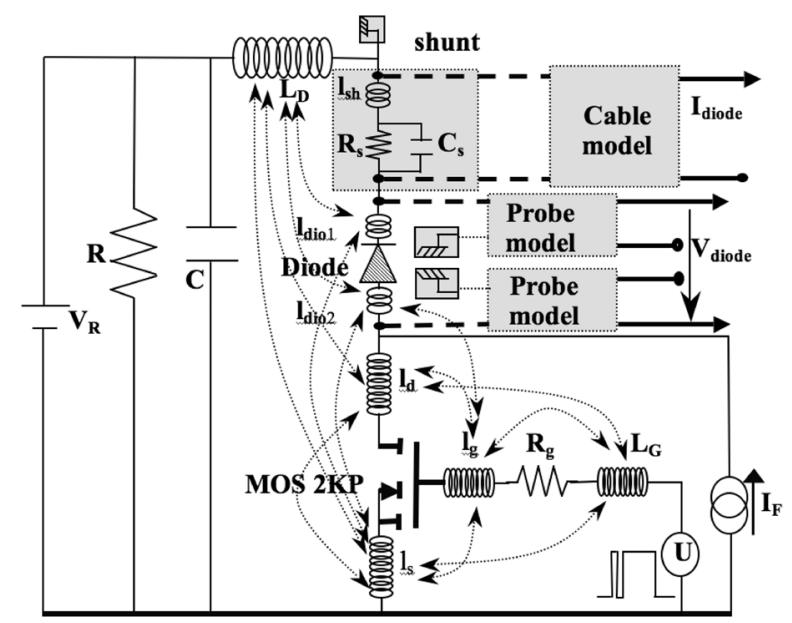

Fig. 2. Experimental and simulated circuits of the switching cell including parasitic interconnection model and sensor models.

Fig. 2. This configuration has been described in detail in [1]. The switching cell main operating conditions are the forward current, $I_{F}$, and the reverse voltage, $V_{R}$. A current and a voltage source impose these conditions, respectively. A low recurrence is considered for the diode operation; thus ambient temperature is considered for the device temperature. The turn-on and turn-off of the MOSFET transistor operate the turn-off and the turn-on of the PiN diode, respectively. The selected MOSFET model is based on the classical SPICE level-2 MOSFET model, available in major circuit simulation software. A wiring parasitic model of the experimental circuit is taken into account by computing an inductance matrix using the commercial software InCA [3]. Thus, interactions between wiring inductors are computed to avoid any effects that would adversely affect the diode model. Probe models are also considered for the same reason.

The accurate simulation of the STTB506D PiN diode might be obtained using numerical simulators such as MEDICI [4] or DESSIS [5], which solve the semiconductor device equations. These simulators are based on finite-element methods (FEM). The DESSIS simulator is used for mixed-mode device/circuit simulations. From electrical and thermal points of view, the power PiN diode is essentially a 1D device. Hence the PiN diode simulation was limited to a 1-D technological modeling. Many equivalent circuit or state-space variable models have been published in the literature [6], [7]. However, most models show a limitation with regard to high-level injection phenomenon; hence turn-off and turn-on transients may not be accurately estimated. Moreover, these analytical models do not offer a clear relation to the diode technological architecture (required for the present study), hence the choice of a finite-element representation.

\section{Comparison of Two Doping Profile Models}

For the design of the PiN diode doping profile, two cases are considered in 1-D: a simple epitaxial doping profile (conventional doping profile model) and a doping profile with a second epitaxy and the $\mathrm{N}_{2}$ step, as in Fig. 3 (an advanced doping profile

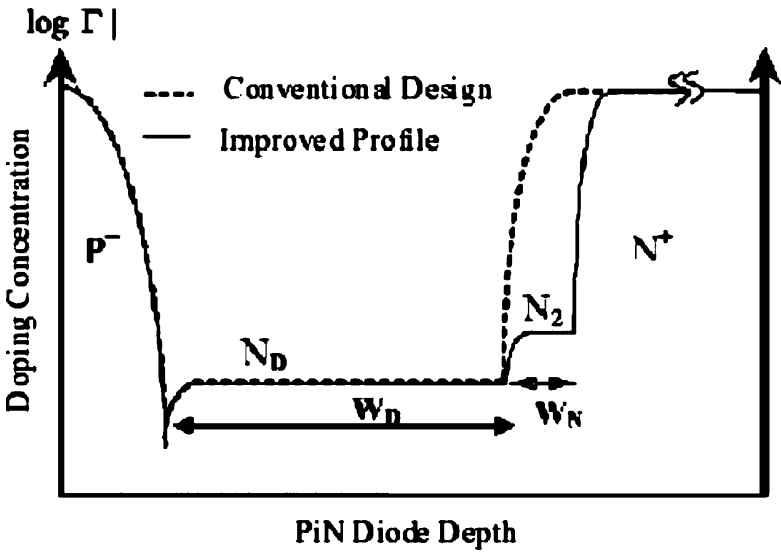

Fig. 3. Two 1-D doping profile models of the PiN diode.

model [2]). Using the main design parameters estimated for the STTB506D diode in [1] and taking into account a conventional doping profile model, a good agreement was obtained between simulated and experimental waveforms of the voltage and current across the device for a forward current, $I_{F}=2 \mathrm{~A}$, for various values of the applied reverse voltage, $V_{R}$ and for various operating temperatures (Fig. 1). The circuit in Fig. 2 is used for all simulations. The conventional doping profile model and the design parameter identification in [1] give a large and satisfactory validity range for the diode turn-off simulation.

However, this agreement between simulation and experiment is no longer valid when the voltage $V_{R}$ is larger than $200 \mathrm{~V}$, particularly at the end of the device turn-off transient, as for $t>t_{3}$, shown in Fig. 4. It may be noted that the simulated voltage waveform across the STTB506D diode presents a non-usual peak at instant $t_{4}$ (for the conventional doping profile model). Fig. 4 shows also that the advanced doping profile model produces a much better estimation of the current and voltage waveforms during the diode turn-off transient. The same design parameter identification procedure is used as for the conventional doping profile model.

The disagreement with the conventional doping profile model is due to the PiN-diode hard-switching during the turn-off transient. Indeed, the FEM simulation of the semiconductor device equations in the low-doped base region of the PiN-diode no longer presents a high concentration of holes to insure conduction $\left(t_{3}<t<t_{4}\right.$ in Fig. 5). This phenomenon is analog to the so-called Kirk effect in the power bipolar transistor. The current continuity has to be satisfied despite the local lack in charge carriers. Therefore the electric field increases locally around the $\mathrm{N}-\mathrm{N}+$ junction to insure the current continuity (zoom in Fig. 6).

The electric field corresponds to an additional over-voltage $V_{\mathrm{RM}}^{a}$, as shown in Fig. 4. The simulated concentration of electrons in this region becomes higher than the doping profile to insure conduction by electron drift. This phenomenon explains the non-usual peak in the voltage waveform. So the FEM simulation shows clearly that to avoid this phenomenon, the doping level close to the $\mathrm{N}-\mathrm{N}^{+}$junction has to be higher in this particular region. Consequently, to obtain a soft-switching PiN diode during the turn-off transient, a doping profile with at least one step $\left(\mathrm{N}_{2}\right)$ is retained. 

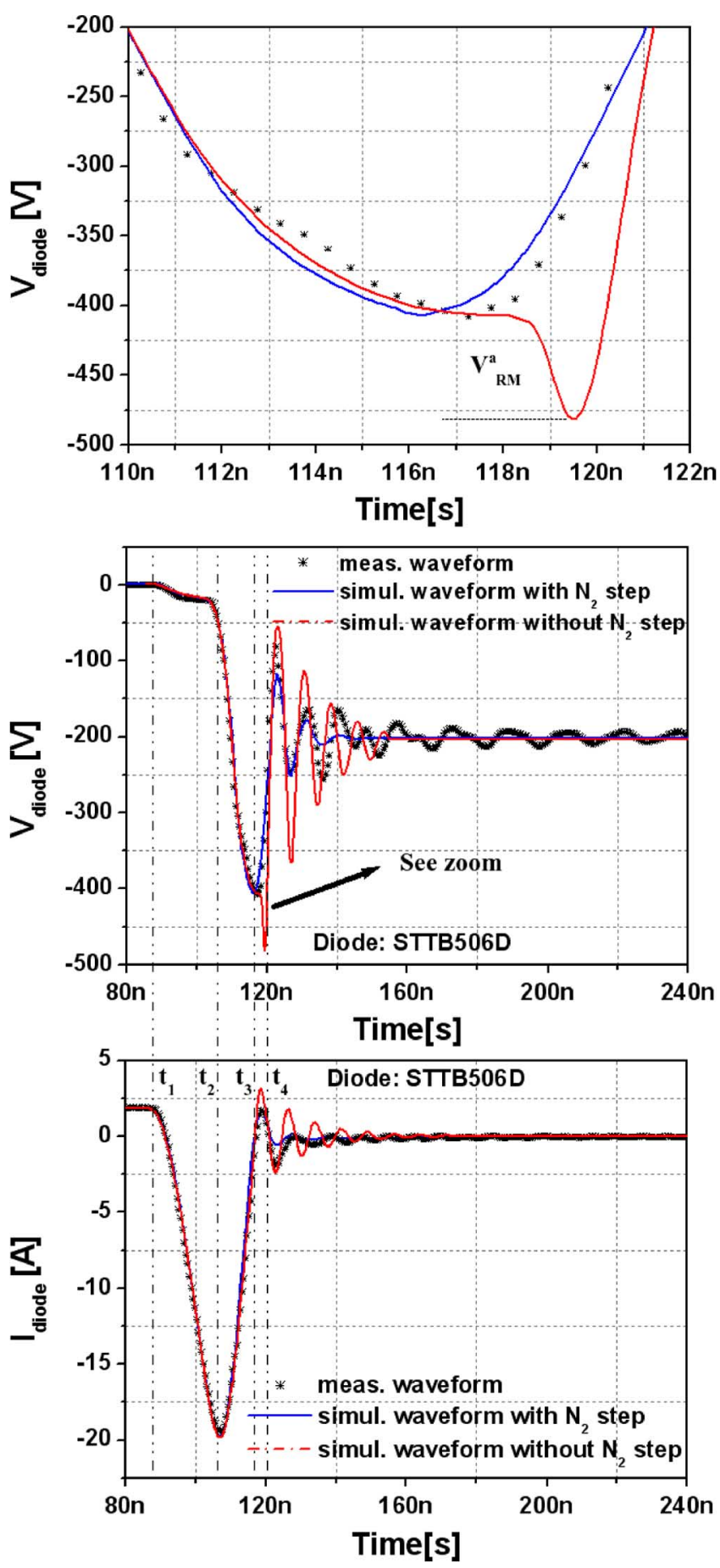

Fig. 4. Measured and simulated waveforms during turn-off of the STTB506D diode considering conventional and advanced technological 1-D models at room temperature.

The advanced doping profile consists of a low-doped base region, of concentration $\mathrm{N}_{D}$, to hold up a high reverse voltage and a thinner region, of concentration $\mathrm{N}_{2}$ slightly larger than $\mathrm{N}_{D}$. The $\mathrm{N}_{2}$ region doping has to be high enough to insure the electron drift, as explained above, but not too much to enable conductivity modulation with high-level injection operating conditions. Using the main design parameters estimated for the STTB506D and taking into account the advanced doping

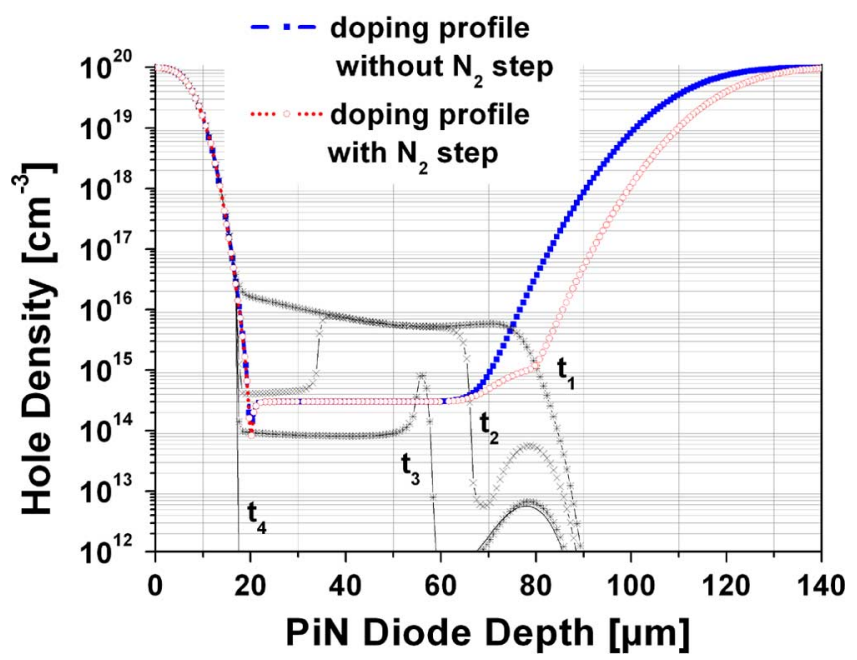

Fig. 5. Hole density related to the doping profile without step of the PiN diode for various instants during its turn-off transient behavior.
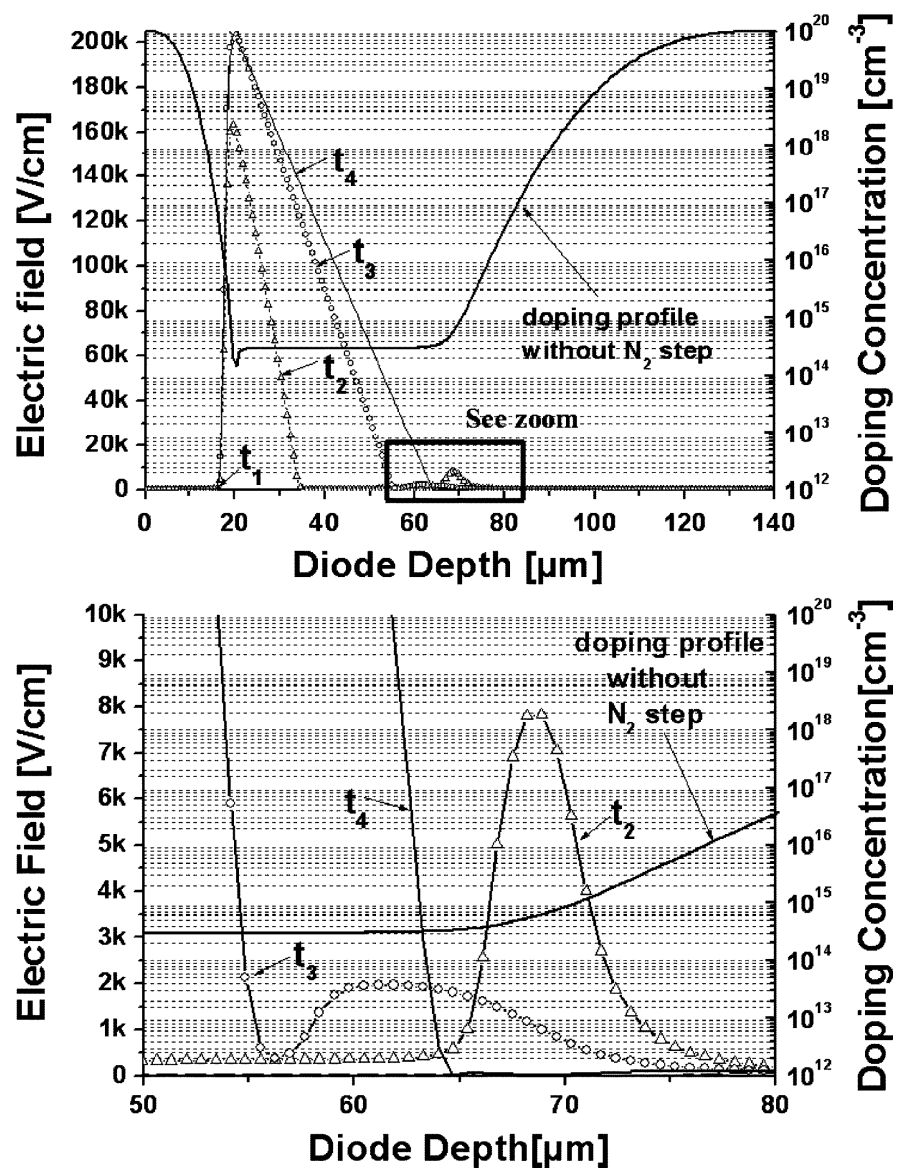

Fig. 6. Electric field related to the doping profile without step of the PiN diode for various instants during its turn-off transient behavior (top). Zoom at the extremity of the diode base region (bottom).

profile model, the current and voltage waveforms across the STTB506D diode during its turn-off transient are simulated and given in Fig. 4 for the parameter set: $W_{N}=15 \mu \mathrm{m}$, $\mathrm{N}_{2}=1.10^{15} \mathrm{~cm}^{-3}$. The $\mathrm{N}_{2}$ region width, $W_{N}$ and its doping concentration, $\mathrm{N}_{2}$, are defined in Fig. 3. When the advanced doping profile model is considered instead of the conventional 
one for the PiN diode, the peak in the simulated voltage waveform across the diode under test does not appear and a good agreement between simulated and experimental voltage and current waveforms is found, as shown in Fig. 4.

A systematic assessment of the validity range of the advanced diode model is to be carried out. This systematic comparison between simulations and measurements should help ascertain if the obtained values of $W_{N}$ and $\mathrm{N}_{2}$ are the best practical values for the STTB506D device. If the validity range is not satisfactory, it is then interesting to question if other epitaxial steps have to be considered at the $\mathrm{N}-\mathrm{N}^{+}$junction of the PiN diode. So far, the authors have shown that the proposed advanced doping profile model brings a significant improvement in accuracy in the case of the PiN diode turn-off simulation and for hard voltage and current conditions. They have successfully applied the advanced doping profile model to various commercial devices.

\section{CONCLUSION}

In a previous work, the authors demonstrated the usefulness of a simple 1-D doping profile for the simulation of the PiN diode transients. The identification of the main design parameters was proposed and a large validity range obtained for the identified models. However a disagreement between simulation and experiment appears in the case of hard operating conditions. This letter has shown the effect of the conventional doping profile model on the simulation of turn-off in these conditions. It is necessary to consider an advanced doping profile for the PiN diode (such as the $\mathrm{N}-\mathrm{N}^{+}$step profile) to obtain better simulation results in hard switching conditions. The simulation results then agree satisfactorily with experimental results. Some improvements are also noted with the automation of the identification procedure of the $\mathrm{WN}$ and $\mathrm{N} 2$ parameters.

\section{REFERENCES}

[1] H. Garrab, B. Allard, H. Morel, K. Ammous, S. Ghedira, A. Amimi, K. Besbes, and J. M. Guichon, "On the extraction of PIN diode design parameters for validation of integrated power converter design," IEEE Trans. Power Electron., vol. 20, no. 3, pp. 660-670, May 2005.

[2] B. Baliga, Modern Power Devices., 2nd ed. Malabar, FL: Krieger Publishing, 1995.

[3] “INCA: User's Guide Manual," INCA Consortium, 1995.

[4] “Medici: User's Guide Manual," Technology Modeling Associates (TMA), 1999, 1999.

[5] “Dessis-ISE Version 9: User's Guide," ISE [Online]. Available: http:// www.ise.com

[6] C. M. Tan and K.-J. Tseng, "Using power diode models for circuit simulations-A comprehensive review," IEEE Trans. Ind. Electron., vol. 46, no. 3, pp. 637-645, Jun. 1999.

[7] Z. Wang, A. T. Bryant, J. Wu, and P. R. Palmer, "Implementation and comparison of power diode models for system simulation," in Proc. IEEE Power Electron. Drives Syst. Conf., 2005, pp. 694-699. 